\title{
Length and GC-biases during sequencing library amplification: A comparison of various polymerase-buffer systems with ancient and modern DNA sequencing libraries
}

\author{
Jesse Dabney and Matthias Meyer \\ Department of Evolutionary Genetics, Max Planck Institute for Evolutionary Anthropology, Leipzig, Germany
}

BioTechniques 52:87-94 (February 2012) doi 10.2144/000113809

Keywords: Ancient DNA; PCR bias; sequencing library amplification; PCR polymerases

Supplementary material for this article is available at www.BioTechniques.com/article/113809

High-throughput sequencing technologies frequently necessitate the use of PCR for sequencing library amplification. PCR is a sometimes enigmatic process and is known to introduce biases. Here we perform a simple amplification-sequencing assay using 10 commercially available polymerase-buffer systems to amplify libraries prepared from both modern and ancient DNA. We compare the performance of the polymerases with respect to a previously uncharacterized template length bias, as well as GC-content bias, and find that simply avoiding certain polymerase can dramatically decrease the occurrence of both. For amplification of ancient DNA, we found that some commonly used polymerases strongly bias against amplification of endogenous DNA in favor of GC-rich microbial contamination, in our case reducing the fraction of endogenous sequences to almost half.

PCR is a ubiquitous and indeed fundamental tool in genetic experimentation. For decades it has allowed rapid in vitro enrichment of targeted loci, increasing the throughput and resolution of many laboratory applications as well as opening new possibilities for genetic analysis (1). PCR is, however, known to be a highly problematic process for the amplification of multiple templates in parallel, where factors such as base composition can have critical effects on experimental results (2). For example, differential template composition can cause allelic dropout in PCR-mediated genotyping (3), or it can cause misrepresentation of pathology associated gene dosage determined by multiplex PCR assays $(4,5)$.

PCR biases can be especially damaging to high-throughput DNA sequencing applications. The most widespread of these technologies require the conversion of DNA or RNA samples into sequencing libraries by attaching two different adaptor sequences to the ends of template molecules. Libraries are only rarely used directly for sequencing (6), but instead are often amplified immediately (7) or after some down-stream processes such as target enrichment by hybridization capture (8). Amplification of sequencing libraries, i.e., amplifying a mixture of templates indiscriminately in parallel, is an enormous challenge and the choice of PCR parameters may strongly determine to which level it is possible to maintain complexity and ensure a representative final product. In fact, it has been shown that library amplification can severely bias the GC-content of sequences $(9,10)$. However, despite extensive use of PCR for the amplification of sequencing libraries, we are only aware of a single study in this direction, which aimed to reduce GC biases in this process (11). In this study, Aird et al. describe an optimized PCR protocol that mitigates a GC-bias introduced during the PCR stage of library preparation. They show that switching polymerases in conjunction with a longer denaturation step and a lower annealing and extension temperature retains the GC-content profile seen before PCR. While satisfactorily overcoming the GC-bias, their investigation was limited to only the GC-bias and a comparison of two polymerases (Phusion $\mathrm{HF}$ and AccuPrime Taq HiFi). Another possible bias, size bias, has yet to be investigated, despite being relevant to applications using samples of limited quantity and/or sequencing technologies supporting longer read lengths, such as Roche/454's Genome Sequencer. Furthermore, to date there has not been a thorough comparison of the capabilities of different polymerases.

In this study we amplify Illumina sequencing libraries prepared from human genomic DNA using 10 different commercially available polymerase/buffer systems and analyze sequence data to determine the effect of these polymerases on fragment length and GC distributions. We also investigate the effect of amplification into the plateau phase of PCR. We then further characterize a subset of the polymerase/ buffer systems with respect to their performance on an ancient DNA library, an extreme application where amplification biases can have severe and deteriorating effects. Ancient DNA libraries are characterized by a distinctive fragmentation pattern, with lengths typically less than $200 \mathrm{bp}$ (12), and low initial quantities made up of a minimum percentage of endogenous DNA often scattered among a high 
background of microbial contamination $(13,14)$. Since these libraries are created from valuable and limited sources, it is often desired to sequence them to exhaustion. Unbiased amplification is particularly important, as an unequal accumulation of a specific template characteristic, such as short fragments or high GC-percentage, can drive up sequencing costs.

\section{Materials and methods \\ DNA Samples}

Two $\mu \mathrm{g}$ of human genomic DNA derived from B-lymphocytes was sheared on a Covaris S2 (Covaris Inc, Woburn, MA) with settings of $10 \%$ duty cycle, intensity 4 and 200 cycles per burst. The fragment library was then visualized with a High Sensitivity DNA chip on a BioAnalyzer (Agilent, Santa Clara, CA) to ensure an appropriate peak and length distribution. Ancient DNA was extracted from $42 \mathrm{mg}$ of a Neandertal toe phalanx from Denisova Cave, Russia (15) using a silica-based extraction technique (16).

\section{Illumina Library Preparation}

Illumina libraries were prepared from both the human and Neandertal samples in accordance with a previously published protocol (17), beginning at the blunt-end repair step, and using the following modifications: (i) For the human sample, fulllength adaptors (P5 and indexed P7) were ligated directly onto all fragments in the adaptor ligation step, thus eliminating the need for amplification with 5'-tailed primers prior to sequencing. (ii) For the Neandertal sample, uracils were excised from the template strands as described elsewhere (18). (iii) For both samples, all purifications steps were done with the MinElute PCR purification kit (Qiagen, Hilden, Germany). Contamination from modern human DNA in the Neandertal extract was determined to be $\sim 2.4 \%$ based on a mitochondrial capture assay described elsewhere (19).

Both libraries were quantified by qPCR as described elsewhere $(17,20)$ and diluted to working concentrations of $\sim 2.0 \times 10^{6}$ molecules/ $\mu$ l for the human DNA library, and $\sim 2.0 \times 10^{5}$ for the Neandertal DNA library.

Modern Human Library Amplification Ten different commercially available polymerases and buffers were used in separate reactions of 15,25 and 40 cycles per polymerase, for a total of 30 distinct amplifications. All reactions were done using $1 \mu \mathrm{L}$ of library as template. As the modern human library already contained
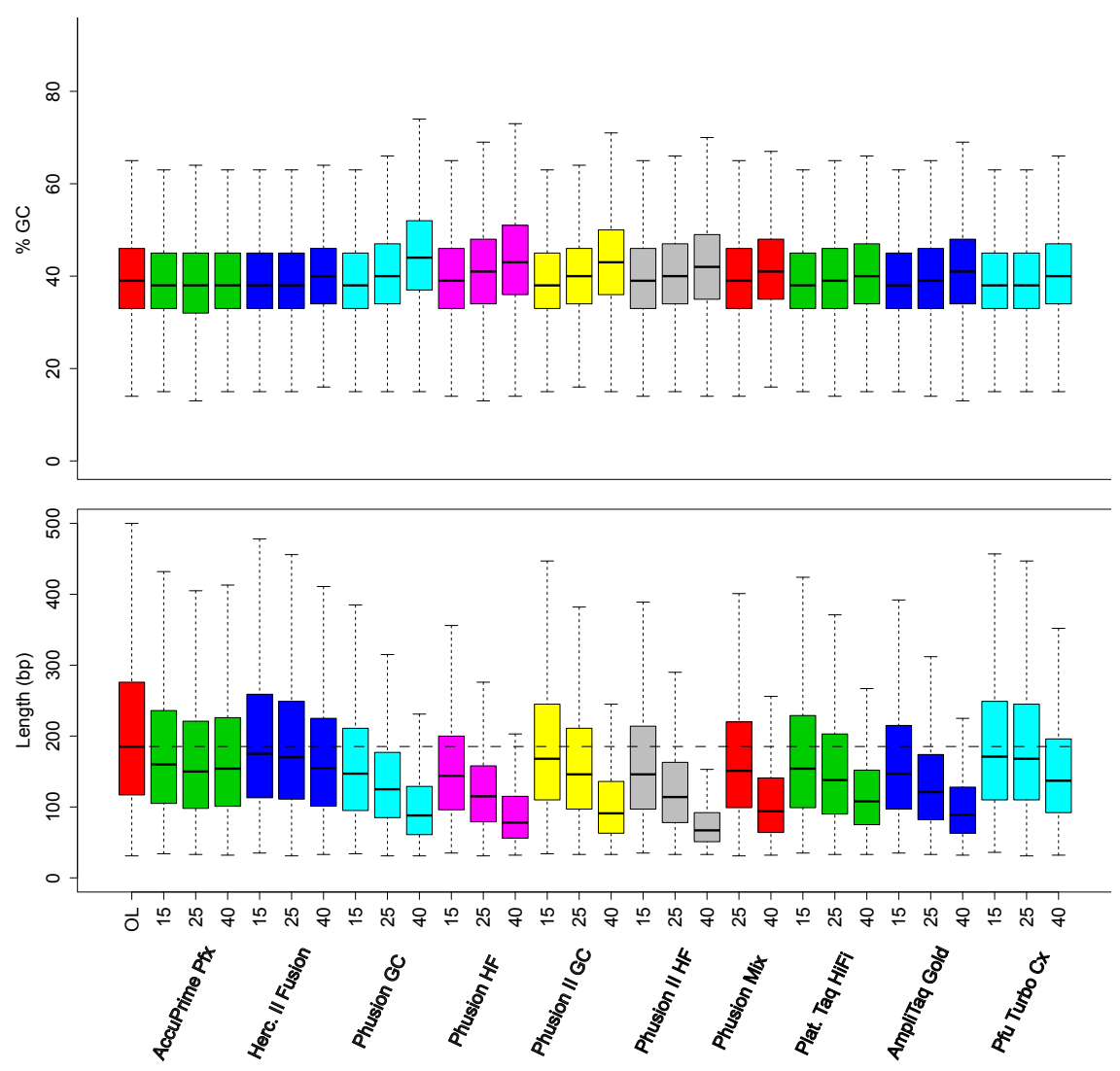

Figure 1. GC-content and Length distributions of a single sequencing library amplified with 10 different polymerase-buffer systems. Distributions are in ascending cycle number order (15, 25 and 40) and color coded by polymerase. Boxes (inter quartile range, IQR) represent the middle $50 \%$ of the data. Median values are denoted by black lines through the IQR, and whiskers extend to I.5 IQR. The original, unamplified library is depicted in the left-most red column. The dotted line across the length plot represents the median value of the original library. Distributions for Phusion Mix 15 cycles were excluded due to insufficient sequence retrieval.

an indexed P7 adaptor, all amplifications were performed with a uniquely indexed P5 primer, making use of a recently described double indexing scheme (21). The amplification scheme is depicted in Supplementary Figure 1A. Cycling was performed on a DNA Engine Dyad (Bio-Rad, Munich, Germany). Reactions were spin-column purified (MinElute PCR purification kit, Qiagen). The following polymerases and manufacturer's conditions were used:

Herculase II Fusion (Agilent, Waldbronn, Germany) - 1x Herculase Buffer, $0.25 \mathrm{mM}$ of each dNTP (Fermentas, St. Leon-Rot, Germany), $0.25 \mu \mathrm{M}$ P5 index primer, $0.25 \mu \mathrm{M}$ P7 primer, $0.5 \mu \mathrm{L}$ Herculase II Fusion polymerase, water to $50 \mu \mathrm{L}$. The thermocycling profile was 2 min at $95^{\circ} \mathrm{C}$, n cycles of $20 \mathrm{~s}$ at $95^{\circ} \mathrm{C}, 20 \mathrm{~s}$ at $60^{\circ} \mathrm{C}$ and $40 \mathrm{~s}$ at $72^{\circ} \mathrm{C}$ followed by a final extension step of $3 \mathrm{~min}$ at $72^{\circ} \mathrm{C}$.

Phusion Hot Start I and II with HF and GC buffers (Finnzymes, Vantaa, Finland) - 1x HF or GC buffer, $0.2 \mathrm{mM}$ of each dNTP (Fermentas), $0.5 \mu \mathrm{M}$ P5 index primer, $0.5 \mu \mathrm{M}$ P7 primer, 2.5 units
Phusion or Phusion II polymerase, water to $50 \mu \mathrm{L}$. The thermocycling profile was 30 s at $98^{\circ} \mathrm{C}$, n cycles of 10 s at $98^{\circ} \mathrm{C}, 20$ s at $60^{\circ} \mathrm{C}$ and $40 \mathrm{~s}$ at $72^{\circ} \mathrm{C}$ with a final extension step of $5 \mathrm{~min}$ at $72^{\circ} \mathrm{C}$.

Phusion High Fidelity Master Mix (Finnzymes) - 1x Phusion Master Mix with HF buffer, $0.5 \mu \mathrm{M}$ P5 index primer, $0.5 \mu \mathrm{M}$ P7 primer, water to $50 \mu \mathrm{L}$. The thermocycling profile was $30 \mathrm{~s}$ at $98^{\circ} \mathrm{C}$, n cycles of $10 \mathrm{~s}$ at $98^{\circ} \mathrm{C}, 20 \mathrm{~s}$ at $60^{\circ} \mathrm{C}$ and $40 \mathrm{~s}$ at $72^{\circ} \mathrm{C}$ with a final extension step of $5 \mathrm{~min}$ at $72^{\circ} \mathrm{C}$.

AmpliTaq Gold (Applied Biosystems, Darmstadt, Germany) - 1x Gold Buffer, $2.5 \mathrm{mM} \mathrm{MgCl}, 0.2 \mathrm{mM}$ of each dNTP (Fermentas), $0.2 \mu \mathrm{M}$ P5 index primer, 0.2 $\mu$ M P7 index primer, 2.5 units AmpliTaq Gold Polymerase, water to $50 \mu \mathrm{L}$. The thermocycling profile was $12 \mathrm{~min}$ at $95^{\circ} \mathrm{C}$, $n$ cycles of $20 \mathrm{~s}$ at $95^{\circ} \mathrm{C}, 30 \mathrm{~s}$ at $60^{\circ} \mathrm{C}$ and $40 \mathrm{~s}$ at $72^{\circ} \mathrm{C}$, and a final extension step of $5 \mathrm{~min}$ at $72^{\circ} \mathrm{C}$.

Platinum Taq High Fidelity (Invitrogen, Darmstadt, Germany) - 1x High Fidelity Buffer, $0.2 \mathrm{mM}$ each dNTP (Fermentas), $2 \mathrm{mM} \mathrm{MgSO}_{4}, 0.2 \mu \mathrm{M}$ P5 index primer, 

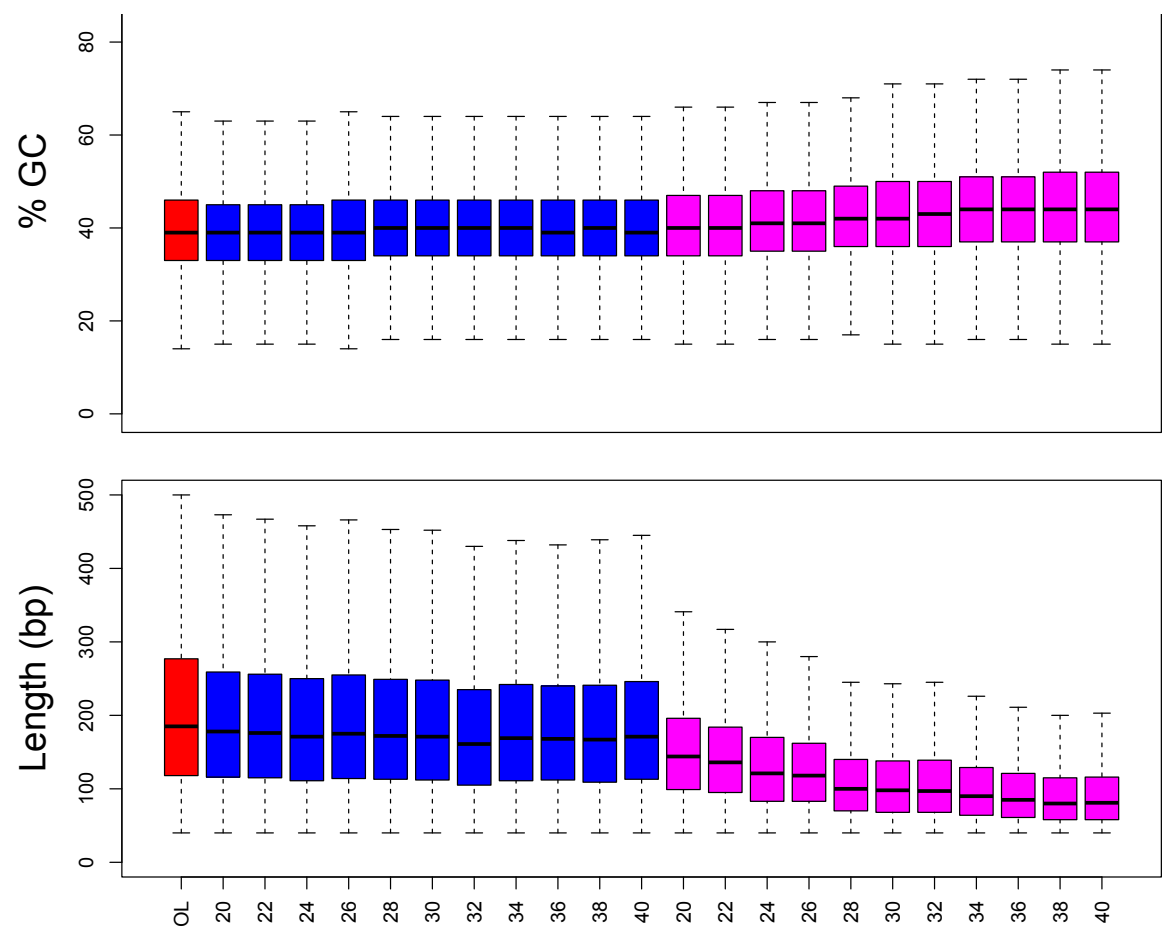

Original Library

Herculase II Fusion

Phusion HF

Figure 2. Higher Resolution GC-content and Length Distributions for a sequencing library amplified with Herculase II Fusion and Phusion HF. The original, unamplified library is depicted in the red column. PCR reactions were performed at 2-cycle intervals from 20 to 40 cycles. Distributions suggest the presence of experimental noise, but corroborate the trends of decreasing length and increasing GC-content as cycle number increases.

$0.2 \mu \mathrm{M}$ P7 primer, 2.5 units Platinum Taq High Fidelity Polymerase, water to $50 \mu \mathrm{L}$. The thermocycling profile was $1 \mathrm{~min}$ at $94^{\circ} \mathrm{C}$, n cycles of 20 s at $94^{\circ} \mathrm{C}, 30$ s at $60^{\circ} \mathrm{C}$ and $1 \mathrm{~min}, 10 \mathrm{~s}$ at $68^{\circ} \mathrm{C}$.

Pfu Turbo Cx Hotstart (Agilent) - 1x Pfu Turbo Cx buffer, $0.2 \mathrm{mM}$ each dNTP (Fermentas), $0.2 \mu \mathrm{M}$ P5 index primer, 0.2 $\mu$ M P7 primer and 2.5 units Pfu Turbo Cx polymerase. The thermocycling profile was $2 \mathrm{~min}$ at $95^{\circ} \mathrm{C}, \mathrm{n}$ cycles of $30 \mathrm{~s}$ at $95^{\circ} \mathrm{C}, 30 \mathrm{~s}$ at $60^{\circ} \mathrm{C}$ and $1 \mathrm{~min}, 10 \mathrm{~s}$ at $72^{\circ} \mathrm{C}$ followed by a final extension step of $10 \mathrm{~min}$ at $72^{\circ} \mathrm{C}$.

AccuPrime Pfx Polymerase (Invitrogen) - 1x AccuPrime reaction mix, $0.3 \mu \mathrm{M}$ P5 index primer, $0.3 \mu \mathrm{M}$ P7 primer and 1.25 units AccuPrime Pfx polymerase. The thermocycling profile was $2 \mathrm{~min}$ at $95^{\circ} \mathrm{C}$, $\mathrm{n}$ cycles of $15 \mathrm{~s}$ at $95^{\circ} \mathrm{C}, 30 \mathrm{~s}$ at $60^{\circ} \mathrm{C}$ and 1 $\min , 10 \mathrm{~s}$ at $68^{\circ} \mathrm{C}$.

Neandertal Library amplification Polymerase and PCR conditions remained the same as in the modern human library amplifications, however Phusion II, Phusion Master Mix and Phusion I with GC buffer were excluded from this assay. Reactions were done in replicates of 6 for 26 cycles per reaction. Each reaction contained uniquely indexed P5 and P7 primers (Supplementary
Figure 1B). All reactions were purified with the MinElute PCR purification kit (Qiagen).

Quantification and pooling of

PCR products

Products amplified from the modern human library for 25 cycles were quantified with a DNA 1000 chip on a BioAnalyzer (Agilent). Amplicons from 15 and 45 cycle reactions had to be quantified differently because of insufficient concentrations or the presence of heteroduplexes. Therefore, one 25-cycle amplicon was taken as representative and a dilution series was created to use as a qPCR standard for quantification of all other amplified libraries from modern human samples. qPCR counts were used to determine dilution factors for each PCR product so that they could be pooled in equimolar ratios for sequencing.

All products amplified from the Neandertal library were quantified on a DNA 1000 chip (Agilent) and pooled in equimolar ratios for sequencing.

Illumina sequencing and sequence analysis

The resulting libraries of pooled PCR product as well as the original, unamplified library were sequenced on separate lanes of 75 cycle double-index paired-end runs on the Illumina GAII platform as described in Kircher et. al. (21) and bases were called with the machine learning algorithm Ibis (22).

For the modern human data, raw sequences were sorted by index and split into forward and reverse sequences and trimmed to $40 \mathrm{bp}$. These sequences were mapped to the human reference genome (hg19 $1000 \mathrm{~g}$ ) using BWA (23). Mate pairs were filtered for mapping quality $\geq$ 30 , and the start and end coordinates were used to extract the putative insert sequence from the reference genome. The resulting sequences were then subjected to a length filter excluding sequences less than $40 \mathrm{bp}$ and more than $500 \mathrm{bp}$, and these sequences were used in the subsequent analysis.

For the Neandertal data, sequences were sorted by index and paired-end reads were merged into single sequences to eliminate adapter sequences from short insert reads (24). The sequences were mapped to the human reference genome (hg19-1000 g) using BWA (23). Quality and size filters were then applied to exclude sequences with a mapping quality of $<30$, and lengths shorter than $35 \mathrm{bp}$. An upper length cutoff of $139 \mathrm{bp}$ was applied by using only merged reads for mapping. Of the remaining sequences, a random subsample of 370,000 sequences was taken from each replicate and used in subsequent analysis.

\section{Results and discussion}

Length and GC biases in Modern Human Sequencing Libraries

In order to analyze the extent of size and base composition biases introduced into sequencing libraries by different polymerases, we set up a simple amplification-sequencing assay. We first prepared a sequencing library from sheared human genomic DNA. By definition this library is unbiased by amplification. We then amplified $\sim 2.0 \times 10^{6}$ molecules of this library using ten commercially available DNA polymerase/buffer systems for 15 , 25 and 40 cycles. The polymerase/buffer systems include AccuPrime Pfx (Invitrogen), Herculase II Fusion (Agilent), Pfu Turbo Cx Hotstart (Agilent), Platinum Taq High Fidelity (Invitrogen), AmpliTaq Gold (Applied Biosystems) and Phusion DNA polymerase (Finnzymes). Since Phusion DNA polymerase is recommended for library amplification in many Illumina library preparation protocols, we tested different versions of this enzyme with alternative buffers supplied by the manufacturer. Post-sequencing analysis involved quality 
filtering and lower and upper size cutoffs of $40 \mathrm{bp}$ and $500 \mathrm{bp}$ respectively.

The resulting length and GC distributions are shown in Figure 1. After sequencing, the original input library had a median length of $185 \mathrm{bp}$ and a median GC-content of 39\%. After 15 cycles of PCR, the median length of all libraries had decreased, the most extreme being both Phusion and Phusion II in HF buffer, with 41 bp and 38 bp decreases, respectively. The smallest shifts were observed in the Herculase II Fusion library (10 bp decrease) and the Pfu Turbo Cx library (14 bp decrease). Changes in the median GC-content for all libraries were within one percentage point of the original unamplified library.

Trends were consistent after 25 cycles. Herculase II Fusion and Pfu Turbo Cx libraries again had the smallest shifts in median lengths (14 bp and 16 bp decrease, respectively) and Phusion and Phusion II HF libraries had the highest (70 bp decrease for both). GC-content for all libraries was again within 1-2 percentage points of the original.

The inter-quartile range (IQR), which represents the middle $50 \%$ of the data, decreased concomitantly with the median in all libraries. Accordingly, Herculase II Fusion libraries posted the smallest reductions after 15 and 25 cycles ( $14 \mathrm{bp}$ and 21 bp), followed by Pfu Turbo Cx (20 bp and $24 \mathrm{bp})$. Phusion and Phusion II HF libraries showed the largest IQR reductions across both cycle numbers ( 80 bp and 74 bp respectively after 25 cycles), indicating not only a reduction, but also a severe narrowing of the length distributions.

Shifts in length and GC-content medians, as well as IQRs after 40 cycles were slightly incongruous with previous trends. Decreases in median lengths and IQRs were exaggerated in some cases. For example, after remaining stable through 25 cycles, the median length of the Pfu Turbo Cx library decreased by $47 \mathrm{bp}$. In addition, the median length of the Phusion II HF library dropped 116 bp and the IQR decreased enough to fall completely outside that of the original library. 40 cycles was assumed to be well into the plateau phase of amplification, so in order to determine if these inconsistencies were the result of PCR plateau effects or experimental noise, we performed a higher resolution assay using only Herculase II Fusion and Phusion HF, the best and worst performing polymerases at 25 cycles. This time amplifications were performed at 2 cycle intervals from 20 to 40 cycles (Figure 2 ). It was determined by qPCR that the libraries entered plateau, i.e., no further increase in copy number,
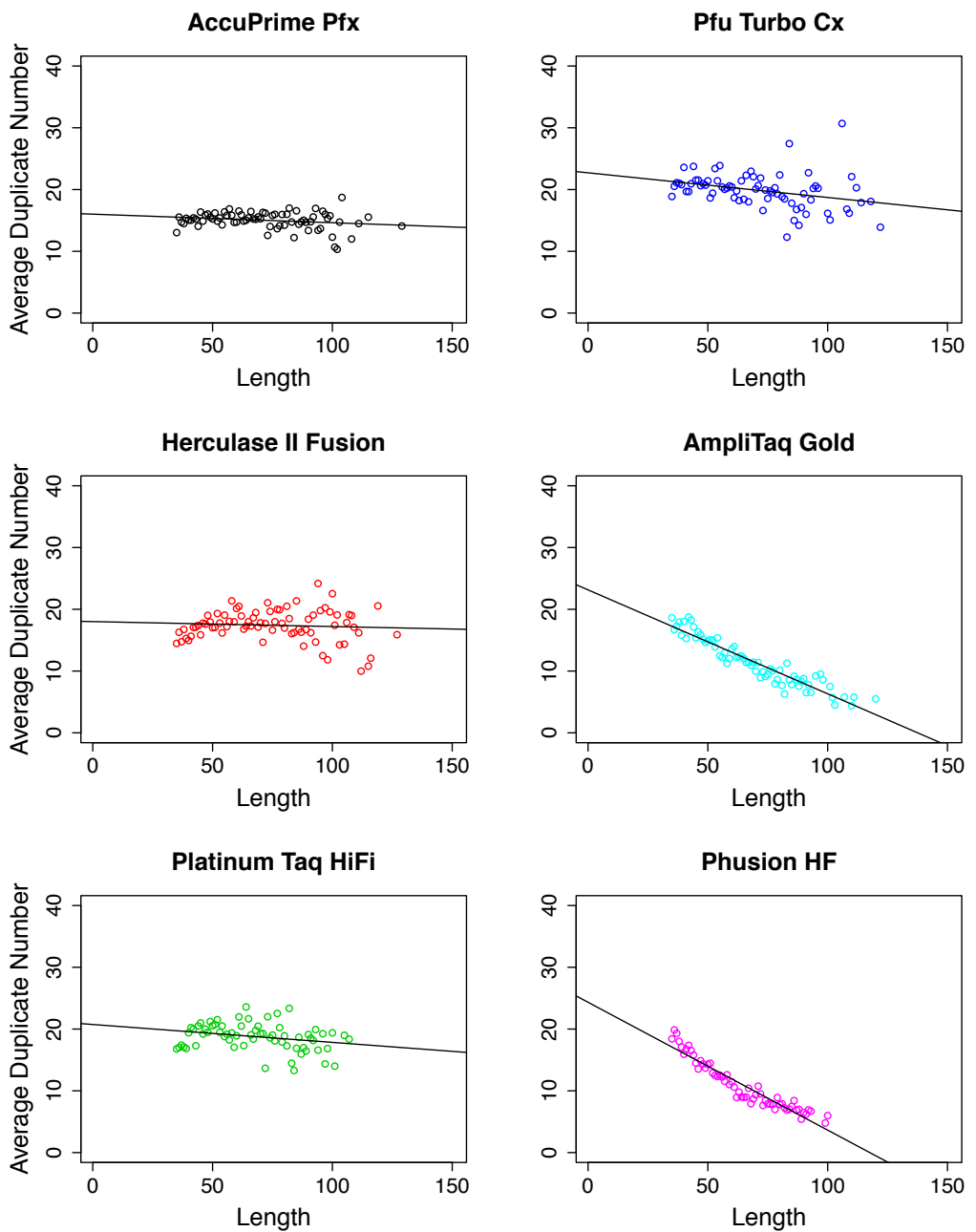

Figure 3. Average Duplicate Number versus Length in ancient DNA sequencing libraries. The average number of duplicate sequences in a given length bin (bin size $=1 \mathrm{bp}$ ) was calculated and plotted against length (for bins with $\geq 10$ observations). Regression lines are plotted as a visual aid.

after 32 and 34 cycles (Herculase II Fusion and Phusion HF, respectively) (data not shown). Our data indicates that biases did not increase upon entering the plateau phase of PCR. Thus the extremes seen after 40 cycles (Figure 1) are mostly due to stochastic amplification in individual reactions. This finding is particularly relevant for the amplification of libraries prior to target enrichment. Applications like hybridization capture on micro-arrays (8) can require up to $20 \mu \mathrm{g}$ of template DNA. In such cases it is difficult to avoid amplifying into plateau. Knowing that cycling into plateau does not magnify these biases will ease future amplifications.

Despite some experimental noise, the data from these amplifications show clear trends. Herculase II Fusion and Phusion HF represent the best and worst performers of the 10 polymerase-buffer systems tested. In libraries amplified with Phusion HF there is a strong negative correlation between length and cycle number $(\mathrm{r}=-0.96, P=$ $1.62 \times 10^{-6}$, Pearson) and a clear positive correlation between GC percentage ( $\mathrm{r}$ $=0.97, P=9.15 \times 10^{-7}$, Pearson). Libraries amplified with Herculase II Fusion were comparatively robust to increasing cycle number. There was no correlation between GC percentage and cycle number and only a weak correlation between length and cycle number ( $\mathrm{r}=-0.66, P=0.02$, Pearson). It is clear from this that a large degree of length and GC-bias in sequencing libraries can be effectively avoided by simply switching the polymerase-buffer system.

\section{Length and GC biases in Ancient DNA Libraries}

Part of the motivation of this study was to eliminate strong length and GC biases 

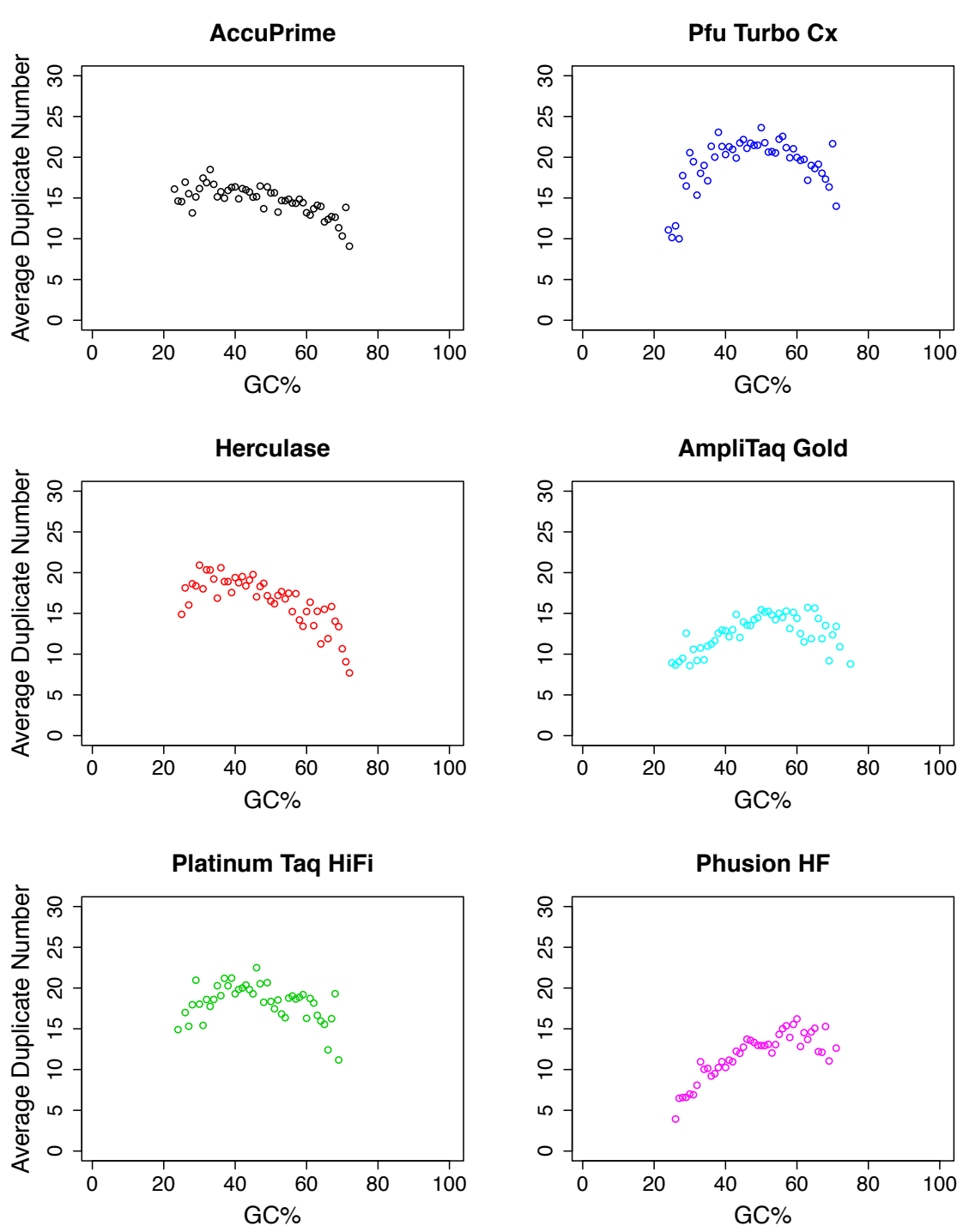

Figure 4. Average Duplicate Number vs GC-content in ancient DNA sequencing libraries. Data are only shown for bins (bin size $=1$ percent) with $\geq 10$ observations.

generated when amplifying ancient DNA libraries. These biases inflate the depth and thus the cost required to thoroughly sequence such libraries. In fact, AmpliTaq Gold and Phusion polymerases, which were found to be among the worst performers in the previous experiments, are commonly used for amplification of aDNA libraries $(25,26)$. The detrimental effects of this become apparent when analyzing data from one such study (25; Supp. Figure 2)

We applied the amplificationsequencing assay to a Neandertal library to further investigate these biases, and to find a suitable polymerase specifically for the amplification of ancient DNA libraries. We pursued a different strategy than done for the modern human library based on heavy oversequencing of a very dilute library and direct counting of the number of duplicate sequences seen from molecules in each length and GC bin. All polymerases from the previous panel were used except for the different Phusion systems. In this case only Phusion HF was used. The input library was treated with uracil-DNA glycosylase and endonuclease VIII prior to amplification to remove deoxyuracils and abasic sites from the template (18). This has the double benefit of increasing downstream sequence accuracy, as well as removing DNA modifications that are known block most polymerases tested here (only AmpliTaq Gold and Pfu Turbo Cx can copy across uracils) (27). Amplifications were performed in replicates of 6 , using a unique indexing primer pair for each reaction. Amplification products were pooled and sequenced on one Illumina GAII lane.
A subset of 370,000 sequences was taken for analysis, corresponding to the minimal number of sequences obtained from each replicate. We observed a striking difference among polymerases in the percentage of sequences that could be mapped to the human genome. Since unmapped sequences putatively come from microbial contamination, this number is usually thought to reflect the fraction of endogenous DNA present in the sample. On average, between 9600 and 10,300 sequences $(2.6 \%-3.0 \%)$ could be mapped to the human reference genome for libraries amplified with AccuPrime Pfx, Herculase II Fusion, Pfu Turbo Cx and Platinum Taq HiFi (Suppl. Table 1). In libraries amplified with AmpliTaq Gold and Phusion HF, only an average of $8373(2.3 \%)$ and $6259(1.7 \%)$ sequences could be mapped, respectively. Thus, amplification with AmpliTaq Gold and Phusion HF results in a drastically lower fraction of endogenous sequences. Interestingly, the difference in percentage of endogenous sequences determined by polymerase choice is even larger than the one recently found between Illumina and Helicos sequencing technologies (13). In that study, Phusion was used for Illumina amplification whereas Helicos sequencing was performed without prior amplification of sample DNA. Our data suggest that PCR bias may fully explain the observed difference.

To investigate the nature of polymerase length bias in ancient DNA samples, we took advantage of the fact that the libraries consist of unique molecules and copies of those molecules produced during amplification. We collapsed the mapped sequences into unique molecules and calculated the number of duplicates per unique molecule in the library (Figure 3). Since unique molecules can only be determined from mapped sequences, this analysis only considers endogenous molecules. Of the 6 polymerases, length and average duplicate number were strongly negatively correlated in AmpliTaq Gold libraries $(\mathrm{r}=0.94, P<$ $2 \times 10^{-6}$, Pearson) and Phusion HF libraries ( $\mathrm{r}=0.95, P<2 \times 10^{-6}$, Pearson). In both libraries there was an approximate 3 -fold reduction in average duplicate number across the range of fragment lengths. Length and duplicate number were only marginally correlated in Pfu Turbo Cx libraries $(\mathrm{r}=0.32, P=0.006$, Pearson) and there was no significant correlation in Platinum Taq HiFi, Herculase II Fusion or AccuPrime Pfx libraries.

While all polymerases exhibited inefficiencies in amplifying library molecules with low $(<30 \%)$ or high $(>70 \%)$ GC-content, biases were smallest with 
AccuPrime Pfx (Figure 4). Phusion HF, and to a lesser extent AmpliTaq Gold, showed a very pronounced bias toward molecules with $>50 \%$ GC, which is in line with the results obtained from modern human DNA above. Since microbial DNA in this ancient sample is characterized by high GC-content ( 64\%), this explains the higher fraction of microbial sequences observed in these libraries. Generally, looking at average duplicate numbers provides a higher resolution of the $\mathrm{GC}$ bias than the analysis performed with modern DNA.

In this study we identified PCR polymerases as a main source of both length and GC-content bias in modern human and short-insert ancient sequencing libraries. Under the parameters tested, Phusion polymerases in HF buffer and AmpliTaq Gold consistently introduced dramatic biases in both types of libraries, while the biases introduced by the other four polymerases are more subtle. For short-insert ancient DNA libraries, AccuPrime Pfx leads to a higher percentage of endogenous sequences while maintaining the length and GC-content profile of the input library. Furthermore, we found no dramatic effect on either length or GC-content bias when amplifying into PCR plateau.

We should note that this was a naïve approach using only the manufacturers' polymerase-buffer systems and suggested parameters. Other factors, such as PCR additives and optimized thermocycling parameters, have been shown to boost performance when dealing with GC-biases (11). In this direction, limiting dilutions and counting PCR duplicates provides a very simple assay tool for detecting PCR biases, and can be used by any lab to characterize and optimize their preferred polymerase.

\section{Acknowledgments}

We would like to thank Susanna Sawyer for providing the Neandertal library, Martin Kircher for data processing, the Sequencing Group for help with sequencing and, finally, Svante Pääbo and the Dept. of Evolutionary Genetics for helpful discussion. This work was supported by the Max Planck Society.

\section{Competing interests}

The authors declare no competing interests.

\section{References}

1. McPherson, M.J. and S.G. Moller. 2006. PCR. Taylor \& Francis Group, New York, NY.
2. Kanagawa, T. 2003. Bias and artifacts in multitemplate polymerase chain reactions (PCR). J. Biosci. Bioeng. 96:317-323.

3. Day, D.J., P.W. Speiser, E. Schulze, M. Bettendorf, J. Fitness, F. Barany, and P.C. White. 1996. Identification of non-amplifying CYP21 genes when using PCR-based diagnosis of 21-hydroxylase deficiency in congenital adrenal hyperplasia (CAH) affected pedigrees. Hum. Mol. Genet. 5:2039-2048.

4. Ogino, S. and R.B. Wilson. 2002. Quantification of PCR bias caused by a single nucleotide polymorphism in SMN gene dosage analysis. J. Mol. Diagn. 4:185-190.

5. Barnard, R., V. Futo, N. Pecheniuk, M. Slattery, and T. Walsh. 1998. PCR bias toward the wild-type k-ras and p53 sequences: implications for PCR detection of mutations and cancer diagnosis. Biotechniques 25:684-691.

6. Kozarewa, I., Z. Ning, M.A. Quail, M.J. Sanders, M. Berriman, D.J. Turner, and N. America. 2009. Amplification-free Illumina sequencing-library preparation facilitates improved mapping and assembly of $(\mathrm{G}+\mathrm{C})$ -biased genomes. Nat. Methods 6:291-295.

7. Margulies, M., M. Egholm, W.E. Altman, S. Attiya, J.S. Bader, L.A. Bemben, J. Berka, et al. 2005. Genome sequencing in microfabricated high-density picolitre reactors. Nature 437:376-380.

8.Hodges, E., M. Rooks, Z.Xuan, A. Bhattacharjee, D. Benjamin Gordon, L. Brizuela, W. Richard McCombie, et al. 2009. Hybrid selection of discrete genomic intervals on custom-designed microarrays for massively parallel sequencing. Nat. Protocols 4:960-974.

9. Mamanova, L., A.J. Coffey, C.E. Scott, I. Kozarewa, E.H. Turner, A. Kumar, E. Howard, et al. 2010. Target-enrichment strategies for next-generation sequencing. Nat. Methods 7:111-118.

10. Sam, L.T., D. Lipson, T. Raz, X. Cao, J. Thompson, P.M. Milos, D. Robinson, et al. 2011. A comparison of single molecule and amplification based sequencing of cancer transcriptomes. PLoS One 6:e17305.

11. Aird, D., M.G. Ross, W.S. Chen, M. Danielsson, T. Fennell, C. Russ, D.B. Jaffe, et al. 2011. Analyzing and minimizing PCR amplification bias in Illumina sequencing libraries. Genome Biol. 12:R18.

12. Pääbo, S. 1989. Ancient DNA: extraction, characterization, molecular cloning, and enzymatic amplification. Proc. Natl. Acad. Sci. USA 86:1939-1943.

13. Orlando, L., A. Ginolhac, and M. Raghavan. 2011. True single-molecule DNA sequencing of a pleistocene horse bone. Genome Res. 21:17051719.

14. Briggs, A.W., U. Stenzel, P.L.F. Johnson, R.E. Green, J. Kelso, K. Prüfer, M. Meyer, et al. 2007. Patterns of damage in genomic DNA sequences from a Neandertal. Proc. Natl. Acad. Sci. USA 104:14616-14621.

15. Gibbons, A. 2011. Who were the Denisovans? Science 333:1084-1087.

16. Rohland, N. and M. Hofreiter. 2007. Ancient DNA extraction from bones and teeth. Nat. Protocols 2:1756-1762.

17. Meyer, M. and M. Kircher. Illumina Sequencing Library Preparation for Highly Multiplexed Target Capture and Sequencing. Cold Spring Harb Protoc. 2010.

18. Briggs, A.W., U. Stenzel, M. Meyer, J. Krause, M. Kircher, and S. Pääbo. 2010. Removal of deaminated cytosines and detection of in vivo methylation in ancient DNA. Nucleic Acids Res. 38:e87.

19. Krause, J., Q. Fu, J.M. Good, B. Viola, M.V. Shunkov, A.P. Derevianko, and S. Pääbo. 2010. The complete mitochondrial DNA genome of an unknown hominin from southern Siberia. Nature 464:894-897.

20. Meyer, M., A.W. Briggs, T. Maricic, B. Höber, B. Höffner, J. Krause, A. Weihmann, et al. 2008. From micrograms to picograms: quantitative PCR reduces the material demands of high-throughput sequencing. Nucleic Acids Res. 36:e5.

21. Kircher, M., S. Sawyer, and M. Meyer. 2011. Double indexing overcomes inaccuracies in multiplex sequencing on the Illumina platform. Nucleic Acids Res. 40:1-8.

22. Kircher, M., U. Stenzel, and J. Kelso. 2009. Improved base calling for the Illumina Genome Analyzer using machine learning strategies. Genome Biol. 10:R83.

23. Li, H. and R. Durbin. 2010. Fast and accurate long-read alignment with Burrows-Wheeler transform. Bioinformatics 26:589-595.

24. Kircher, M., P. Heyn, and J. Kelso. 2011. Addressing challenges in the production and analysis of Illumina sequencing data. BMC Genomics 12:382.

25. Reich, D., R.E. Green, M. Kircher, J. Krause, N. Patterson, E.Y. Durand, B. Viola, et al. 2010. Genetic history of an archaic hominin group from Denisova Cave in Siberia. Nature 468:1053-1060.

26. Rasmussen, M., Y. Li, S. Lindgreen, J.S. Pedersen, A. Albrechtsen, I. Moltke, M. Metspalu, et al. 2010. Ancient human genome sequence of an extinct Palaeo-Eskimo. Nature 463:757-762.

27. Heyn, P., U. Stenzel, A.W. Briggs, M. Kircher, M. Hofreiter, and M. Meyer. 2010. Road blocks on paleogenomes--polymerase extension profiling reveals the frequency of blocking lesions in ancient DNA. Nucleic Acids Res. 38:e161.

Received 28 October 2011; accepted 16 December 2011.

Address correspondence to Jesse Dabney, Max Planck Institute for Evolutionary Anthropology, Department of Evolutionary Genetics, 04103 Leipzig, Germany. Email: jesse_dabney@eva. mpg.de

To purchase reprints of this article, contact: biotechniques@fosterprinting.com 